\title{
Gene expression profiling analysis reveals that the long non-coding RNA uc.412 is involved in mesangial cell proliferation
}

\author{
MINYI YU ${ }^{1,2^{*}}$, ZHENG GUAN $^{1,3^{*}}$, SHANWEN LI $^{1^{*}}$, XIANLI WEN $^{1}$, HUIMIN SHI $^{1}$, GAOTING QU $^{1}$, \\ XIAOYU LU ${ }^{1}$, XIANYI ZHU ${ }^{1}$, BIN WANG ${ }^{4}$, QIHUA FENG $^{2}$, WEIHUA GAN ${ }^{1}$ and AIQING ZHANG ${ }^{1}$ \\ ${ }^{1}$ Department of Pediatric Nephrology, The Second Affiliated Hospital of Nanjing Medical University, \\ Nanjing, Jiangsu 210003; ${ }^{2}$ Department of Rheumatology, Children's Hospital of Soochow University, Suzhou, Jiangsu 215000; \\ ${ }^{3}$ Department of Pediatrics, Yangzhou Maternal and Child Care Service Center, Yangzhou, Jiangsu 225000; \\ ${ }^{4}$ Institute of Nephrology, Zhong Da Hospital, Southeast University, Nanjing, Jiangsu 210000, P.R. China
}

Received March 24, 2019; Accepted August 8, 2019

DOI: $10.3892 / \mathrm{mmr} .2019 .10753$

\begin{abstract}
Hyperproliferation of mesangial cells (MCs) is the central pathological feature observed in certain human renal diseases. Furthermore, the long non-coding RNA uc. 412 is regulated by transforming growth factor $\beta 1$ in mesangial cells in vitro. The present study aimed to investigate whether uc. 412 serves a role in renal fibrosis and whether it may be considered as a therapeutic target in mesangial proliferative kidney diseases. The results demonstrated that uc. 412 overexpression significantly increased $\mathrm{MC}$ proliferation. The transcriptional profile of MCs overexpressing uc. 412 was assessed by RNA sequencing. A total of 462 up- and 843 downregulated genes were identified (Ifold changel $\geq 1.5$ ), and reverse transcription-quantitative PCR was used to determine the expression of these differentially expressed genes (DEGs). Subsequently, the potential function of these DEGs was determined by bioinformatics analyses. The results indicated that these DEGs were involved in numerous signaling pathways associated with MC proliferation. The downstream association between upand downregulated genes was constructed via the STRING database. The protein-protein interaction network indicated that serpin family E member 1 and matrix metallopeptidase 3 may be hub proteins. In conclusion, the present study provided novel insight into the role of uc.412 in MC proliferation, which may aid in the development of novel treatment for mesangial proliferative kidney diseases.
\end{abstract}

Correspondence to: Professor Aiqing Zhang, Department of Pediatric Nephrology, The Second Affiliated Hospital of Nanjing Medical University, 262 Zhongshan North Road, Nanjing, Jiangsu 210003, P.R. China

E-mail: njaiqing@njmu.edu.cn

*Contributed equally

Key words: glomerulonephritis, nephritis, transforming growth factor $\beta 1$, membranoproliferative, hyperplasia

\section{Introduction}

The proliferation of mesangial cells (MCs) is a characteristic feature of glomerular diseases, including lupus nephritis, IgA nephropathy, membranoproliferative glomerulonephritis and diabetic nephropathy (1). Furthermore, in animal models of nephritis, MC proliferation often occurs before mesangial sclerosis and the glomerulosclerosis, and is associated with the increase in extracellular matrix synthesis (2). Regulation of MC proliferation may therefore represent a potential treatment option for numerous kidney diseases (3). Transforming growth factor (TGF)- $\beta 1$ is a cytokine that promotes fibrosis in chronic renal diseases, and may trigger and regulate various types of pathophysiological process. For example, TGF- $\beta$ contributes to glomerular extracellular matrix accumulation by stimulating MCs to produce type I, III and IV collagen, laminin, fibronectin and heparan sulphate proteoglycans, as well as by inhibiting matrix degradation, which contributes to the development of glomerulosclerosis (4). However, TGF- $\beta 1$ also has anti-inflammatory properties in certain pathological conditions. For example, TGF- $\beta$ blockade increased kidney inflammation, which was characterized by the upregulation of MCP1, proinflammatory chemokines and the infiltration of monocytes/macrophages in the kidneys (5). Considering these situations, TGF- $\beta 1$ may not be an optimal therapeutic target for kidney disease (5). It is therefore crucial to explore the TGF- $\beta 1$-associated mechanisms in order to identify its therapeutic targets.

Long non-coding (lnc) RNAs are defined as transcripts of $>200$ nucleotides in length that are not translated into proteins (6). Compared with protein-coding genes, IncRNAs are more conserved and specific to organs, tissues, cell types, developmental stages and disease conditions. In addition, lncRNAs are considered as better therapeutic targets in kidney diseases compared with protein-coding transcriptomes (7). It has been reported that IncRNAs may be more responsible for the early development of diabetic nephropathy compared with protein-coding genes (8). For example, the lncRNA LINC01619 contributes to the development of diabetic nephropathy, as it can regulate microRNA (miR)-27a/forkhead box protein $\mathrm{O} 1$ and endoplasmic reticulum stress, resulting 
in podocyte injury (9). Furthermore, lncARSR was reported to promote the expression of AXL receptor tyrosine kinase and c-MET in renal cell carcinoma cells by competitively binding to $\mathrm{miR}-34 / \mathrm{miR}-449$, which results in cell resistance to sunitinib (10). IncRNAs may therefore represent potential therapeutic targets in kidney diseases.

Our previous study revealed that IncRNA uc.412 is differentially expressed in TGF- $\beta 1$-treated MCs (11). In the present study, in order to elucidate the downstream of roles of IncRNA uc.412, the gene expression profiles in MCs overexpressing lncRNA uc.412 were determined using high-throughput sequencing analysis and verified by reverse-transcription-quantitative (RT-q) PCR. Bioinformatics analysis of the identified differentially expressed genes (DEGs) was achieved to predict the function of uc.412. Results from the present suggested an association between uc.412 and kidney diseases. These data may aid our understanding of the role of uc.412 in mesangial cells and in elucidating the mechanism involved in abnormal MC proliferation.

\section{Materials and methods}

Cell culture. The rat mesangial cell line RMC (cat. no. ATCC CRL-2573) was purchased from the American Type Culture Collection. RMCs were cultured in DMEM (HyClone; GE Healthcare Life Sciences) containing 10\% FBS (Crystalgen, Inc.) and $1 \%$ penicillin/streptomycin (Gibco; Thermo Fisher Scientific, Inc.) and placed at $37^{\circ} \mathrm{C}$ in a humidified incubator containing $5 \% \mathrm{CO}_{2}$. The Medical Ethics Committee of the Second Affiliated Hospital of Nanjing Medical University approved the protocol of this study. The proliferation of MCs is a characteristic feature of glomerular diseases. The TGF- $\beta$ signaling pathway is the main pathway for MC proliferation. The cells were treated with TGF- $\beta 1$ at a concentration of $10 \mathrm{ng} / \mathrm{ml}$ for $24 \mathrm{~h}$ and then MCs were used in further experiments to detect the expression of IncRNA uc.412 (11).

Cell transfection. MCs were transfected with uc.412 plasmid (oeuc.412; VectorBuilder) or empty vector control (oeVec) with Lipofectamine $2000^{\circledR}$ (Invitrogen; Thermo Fisher Scientific, Inc.). Briefly, $2.5 \mu \mathrm{g}$ plasmid was diluted into $125 \mu 1$ Opti-MEM serum-free medium (Gibco; Thermo Fisher Scientific, Inc.), and $6 \mu 1$ Lipofectamine 2000 was diluted with $125 \mu 1$ Opti-MEM serum-free medium and incubated room temperature for $5 \mathrm{~min}$. The two solutions were incubated at room temperature for $20 \mathrm{~min}$ and then added to the cells seeded in 6-well plates at a density of $2 \times 10^{5}$ cells/well. Culture medium was replaced after $8 \mathrm{~h}$. After $24 \mathrm{~h}$, puromycin was used to select cells that were puromycin resistant following transfection. After a further $24 \mathrm{~h}$, these cells were used for further experiment. The transfection efficacy was detected by RT-qPCR.

Cell proliferation assay. Transfected cells were seeded in 96-well plates at the density of $1 \times 10^{4}$ cells/well in sextuplicate and cultured in $100 \mu \mathrm{l}$ DMEM containing 10\% FBS At $0,24,48$ and $72 \mathrm{~h}$ following seeding, $10 \mu \mathrm{l}$ Cell Counting Kit-8 reagent (Dojindo Molecular Technologies, Inc.) was added in each well. Following 30 min incubation, the absorbance was measured at $450 \mathrm{~nm}$ using a microplate reader. The relative cell proliferation rate was normalized to the optical density value at $0 \mathrm{~h}$.

Total RNA extraction and RT-qPCR. TRIzol ${ }^{\circledR}$ (Thermo Fisher Scientific, Inc.) was used to extract total RNA from MCs according to the manufacturers' instructions. Total RNA $(500 \mathrm{ng})$ was transcribed into cDNA using PrimeScript ${ }^{\mathrm{TM}}$ RT Reagent kit (Takara Bio, Inc.), according to the manufacturers' instructions. qPCR analysis was performed with the ABI StepOne Real-Time PCR system (Applied Biosystems; Thermo Fisher Scientific, Inc.) using the SYBR ${ }^{\circledR}$ Premix Ex $\mathrm{Taq}^{\mathrm{TM}}$ kit (Takara Bio, Inc.) and the following thermocycling conditions: $95^{\circ} \mathrm{C}$ for $30 \mathrm{sec}$, followed by 40 cycles of $95^{\circ} \mathrm{C}$ for $5 \mathrm{sec}$ and $60^{\circ} \mathrm{C}$ for $30 \mathrm{sec}$. StepOne software v2.0 (Applied Biosystems; Thermo Fisher Scientific, Inc.) was used to analyze the data based on the $2^{-\Delta \Delta \mathrm{Cq}}$ method and GAPDH was used as the internal control for normalization (12). The primer sequences are listed in Table SI.

High-throughput sequencing. Total RNA was extracted as aforementioned and processed by oligo DT enrichment (rRNA removed), and KAPA Stranded RNA-Seq Library Prep kit (Illumina, Inc.) was used to construct an RNA library. In the library construction process, a chain-specific RNA sequencing library was constructed using double-stranded cDNA synthesis using the dUTP method and subsequently high-fidelity PCR polymerases (13). The quality of the constructed library was verified by the Agilent 2100 Bioanalyzer (Agilent Technologies, Inc.) and quantified by RT-qPCR. Library sequencing was performed on an Illumina HiSeq 4000 (Illumina, Inc.), using $\mathrm{NaOH}$ base denaturing single-strand generation, Illumina flow cell in situ amplification and 150 double-end cycle sequencing. Image processing and base recognition was performed using Solexa Pipeline software version 1.8. The FastQC software (version 0.11.5; Babraham Bioinformatics; Babraham Institute) was used to assess the sequencing quality of the reads and remove joints. The library was compared to the reference genome using Hisat2 software (version 2.1.; http://ccb.jhu.edu/software/hisat2/faq.shtml). StringTie software (version v1.3.3; http://ccb.jhu.edu/software/stringtie/) was used to estimate the transcript abundance. $\mathrm{R}$ software Ballgown was used to determine fragments per kilobase of exon per million fragments mapped at the gene level and at the transcript level and to screen the DEGs between samples. DEGs with $\mid \mathrm{FCl} \geq 1.5$ were highlighted; where FC is fold change.

Bioinformatics analysis. Gene Ontology (GO) clustering and Kyoto Encyclopedia of Genes and Genomes (KEGG; http://www.kegg.jp) pathway analysis were performed on all DEGs identified following RNA sequencing. GO enrichment analysis of DEGs was retrieved using the DAVID database (http://david.abcc.ncifcrf.gov) $(14,15)$. KEGG pathway enrichment analysis and results were visualized using Cytoscape (http://cytoscape.org).

Protein-protein interaction (PPI) network and downstream effects of uc.412. To determine the downstream effects of uc. 412 on the proliferation of MC, the STRING database (http://www.string-db.org) was used to analyze the PPI 

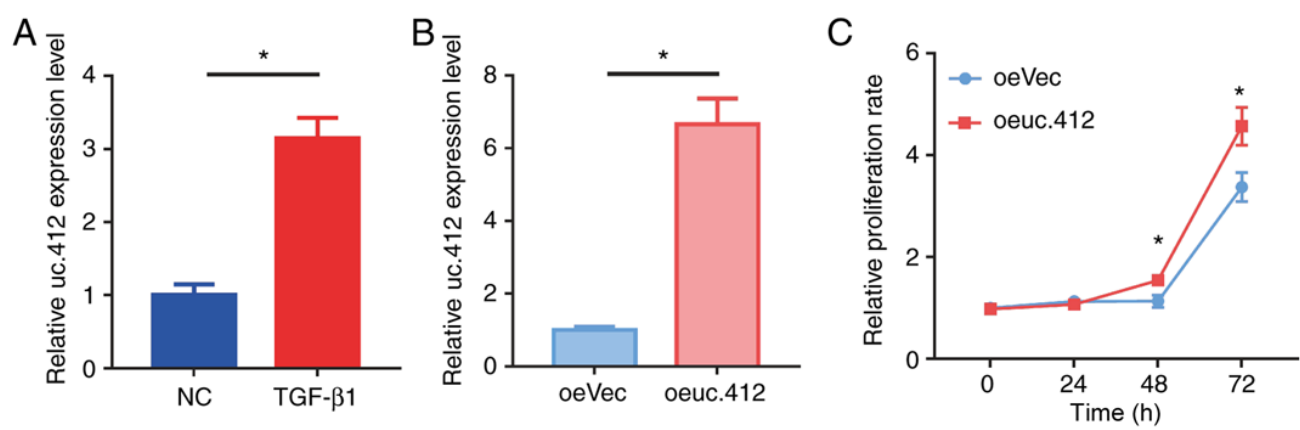

Figure 1. uc.412 overexpression significantly increased MC proliferation. (A) Relative uc.412 expression level in MCs was detected by reverse transcription-quantitative PCR and the results demonstrated that uc.412 was significantly upregulated in TGF- $\beta 1$-treated MCs compared with NC group. Data are presented as the mean $\pm \mathrm{SD}$. Each experiment was performed three times and with five replicates; ${ }^{*} \mathrm{P}<0.05$. (B) Confirmation of successful establishment of uc.412-overexpressing MCs. Data are presented as the mean \pm SD. Each experiment was performed three times and with five replicates; ${ }^{*} \mathrm{P}<0.05$. (C) Proliferation of oeuc.412 MCs is increased compared with the oeVec group. Cell proliferation was detected every $24 \mathrm{~h}$ using the Cell Counting Kit-8. Data are presented as the mean \pm SD. Each experiment was performed three times and in sextuplicate. ${ }^{*} \mathrm{P}<0.05$. LncRNA, long non-coding RNA; MCs, mesangial cells; NC, negative control; oe, overexpressing; TGF- $\beta 1$, transforming growth factor $\beta 1$; uc.412, long non-coding RNA uc.412; vec, vector.

networks of DEGs. The cut-off criterion was set as a combined PPI score of $>0.4$.

Statistical analysis. SPSS 17.0 software (SPSS, Inc.) was used to conduct the statistical analyses. Student's t-test was used to compare two groups. Results were presented as the mean \pm the SD. Each experiment was repeated at least three times. $\mathrm{P}<0.05$ was considered to indicate a statistically significant difference.

\section{Results}

MC proliferation is significantly increased following uc.412 overexpression. RT-qPCR results demonstrated that uc.412 was upregulated in TGF- $\beta 1$-treated MCs $(\mathrm{P}<0.05$; Fig. $1 \mathrm{~A})$. Furthermore, uc.412 expression level was significantly increased in oeuc.412 MCs compared with Control oeVec MCs $(\mathrm{P}<0.05$; Fig. 1B). In addition, the proliferation rate of MCs overexpressing uc.412 was significantly increased after 48 and $72 \mathrm{~h}$ compared with oeVec-transfected MCs $(\mathrm{P}<0.05$; Fig. 1C).

DEG analysis. Following oeuc.412 transfection, a total of 1,305 DEGs were identified from RNA sequencing, of which 462 were upregulated and 843 were downregulated. The scatter plot of the expression level of the DEGs between oeuc.412-transfected group and oeVec-transfected control group is presented in Fig. 2A, and the differences among the top 50 DEGs in each group are highlighted in the heatmaps in Fig. 2B. The full lists of up- and downregulated genes are presented in Tables SII and SIII, respectively.

To verify the DEGs detected by RNA sequencing, nine genes (16) were randomly selected and their mRNA expression levels were examined by RT-qPCR in oeuc.412- and oeVec-transfected MCs; these included five upregulated genes [phospholipase A2 group IIA (PLA2G2A), integrin subunit a6 (ITGA6), fms related tyrosine kinase 1 (FLT1), serpin family E member 1 (SERPINE1) and cd55], and four downregulated genes [kelch-like family member 13 (KLHL13), argonaute RISC component 1 (AGO1), matrix metallopeptidase 3 (MMP3) and tumor necrosis factor receptor superfamily member 1B (TNFRS1B)]. The results demonstrated that the expression levels of PLA2G2A, ITGA6, FLT1, SERPINE1 and CD55 were significantly increased in MCs overexpressing uc.412 compared with control cells (Fig. 3A), whereas KLHL13, AGO1, MMP3 and TNFRS1B expression levels were significantly decreased in oeuc.412 compared with oeVEC MCs (Fig. 3B), which was consistent with the RNA sequencing results. This data identified an interlaced transcript network associated with uc.412.

GO clustering of DEGs. GO clustering was performed on the 1,305 DEGs and included the biological processes, the cellular components and the molecular function categories (Fig. 4). The top nine biological processes associated with the identified DEGs that were identified included 'extracellular matrix organization', 'positive regulation of cell proliferation', 'epithelial to mesenchymal transition', 'negative regulation of protein kinase activity' and 'angiogenesis' (Fig. 4A). The cellular components identified included 'cytosol', 'cytoplasm', 'extracellular matrix', 'cytoskeleton' and 'proteinaceous extracellular matrix' (Fig. 4B). The molecular functions identified included 'protein binding', 'sequence-specific DNA binding', 'extracellular matrix binding', 'protein kinase inhibitor activity' and ‘transcriptional activator activity' (Fig. 4C).

KEGG pathway analysis of DEGs. KEGG pathway analysis was performed on the identified DEGs. The pathways identified included 'p53 signaling pathway', 'Notch signaling pathway', 'MAPK signaling pathway', 'PI3K-Akt singling pathway', 'Cytokine-cytokine receptor interaction', 'HTLV-1 infection', 'AGE-RAGE signaling pathway in diabetic complications' and 'TNF signaling pathway' (Fig. 5). The results indicated that the DEGs associated with uc.412 may be associated with several signaling pathways and make up a complex network.

PPI network and downstream effects of uc.412. The STRING database was used to predict the putative downstream functional genes of uc.412. The results demonstrated that SERPINE1 interacted with connective tissue growth factor (CTGF), hepatocyte growth factor (HGF), FLT1, interleukin 6 (IL6), prostaglandin G/H synthase 2 (PTGS2), tissue-type plasminogen activator (PLAT) and insulin-like growth 
A

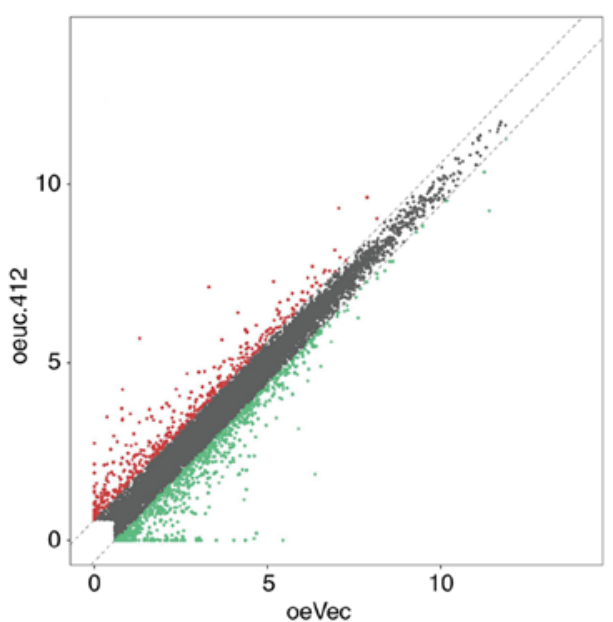

B

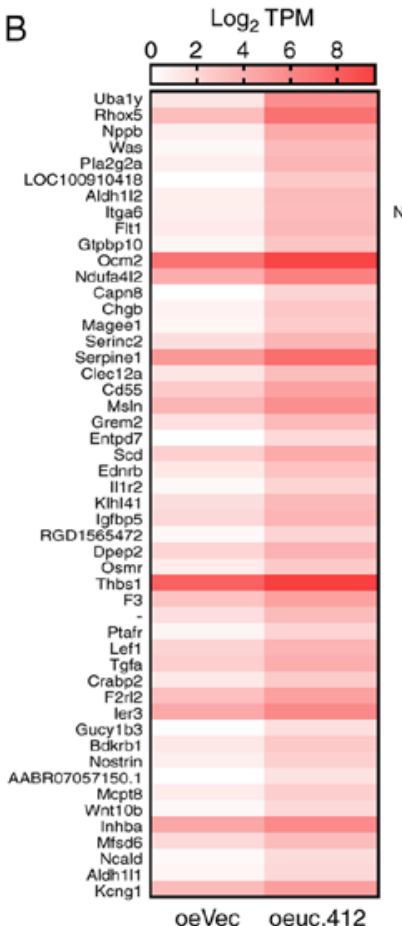

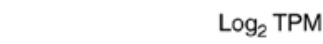

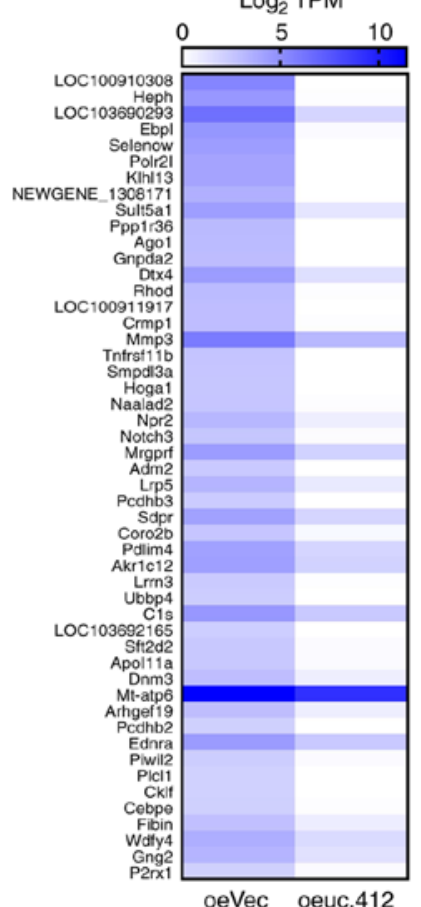

Figure 2. Identification of DEGs between oeuc.412 and oeVec MCs (A) Scatter plot used to determine gene expression variations between uc.412-overexpressing MCs and control MCs. Expression levels were detected and normalized as tag counts per million of TPM. In the scatter-plot, values on $\mathrm{x}$ and $\mathrm{y}$ axes represent the averaged TPM values of each group ( $\log _{2}$ scaled). Red dots represent upregulated genes, and green dots represent downregulated genes, identified as changes of $>1.5$ fold change between the two comparison groups; gray dots indicate genes that were not differentially expressed. (B) DEGs between oeuc.412 MCs and oeVEC negative control. The top 50 upregulated genes and 50 downregulated genes with the highest difference in expression levels. In the left figure, red represent relatively high expression, and white represents relatively low expression. In the right figure, blue represents relatively high expression and white represent relatively low expression. DEGS, differentially expressed genes; MCs, mesangial cells; oe, overexpressing; uc.412, long non-coding RNA uc.412; vec, vector; TPM, total aligned tRNA reads.

factor-binding protein 5 (IGFBP5) in the network of the upregulated genes (Fig. S1A), and, therefore, may be a hub protein. MMP3 interacts with TNFRS1B, ETS domain-containing protein ELK4 (ELK4), integrin subunit $\beta$-like 1 (ITGBL1), endothelin-1 receptor (EDNRA) and cysteine-rich secretory protein 1 (CRISP1) (Fig. S1B).
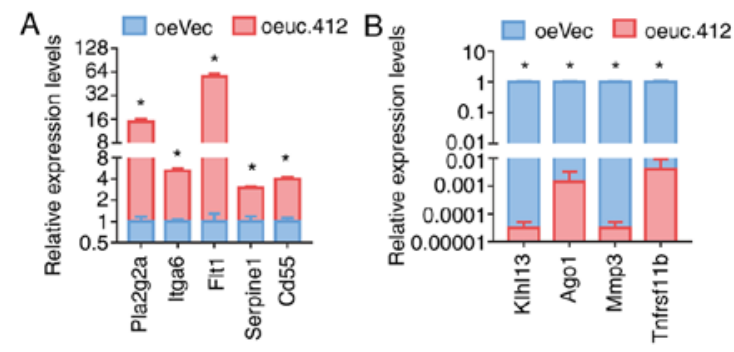

Figure 3. Reverse transcription-quantitative PCR confirmation of the mRNA expression levels of the identified differentially expressed genes. The data indicated that (A) PLA2G2A, ITGA6, FLT1, SERPINE1 and CD55 expression levels were significantly increased, and (B) KLHL13, AGO1, MMP3 and TNFRS1B expression levels were significantly decreased in oeuc.412transfected MCs compared with oeVec-transfected MCs. Each experiment was performed three times and with five replicates; $* \mathrm{P}<0.05$. AGO1, argonaute RISC component 1; FLT1, fms related tyrosine kinase 1; ITGA6, integrin subunit alpha 6; KLHL13, kelch-like family member 13; MMP3, matrix metallopeptidase 3; oe, overexpressing; PLA2G2A, phospholipase A2 group IIA; SERPINE1, serpin family E member 1; TNFRS1B, tumor necrosis factor receptor superfamily member $1 \mathrm{~B}$; uc.412, long non-coding RNA uc.412; vec, vector.

\section{Discussion}

TGF- $\beta 1$ serves a crucial role in promoting fibrosis in tissue (4). Since antifibrotic therapy consisting in targeting TGF- $\beta 1$ to treat renal fibrosis can lead to dysregulation of other normal physiological processes (4), it is necessary to understand the specific downstream mechanisms of TGF- $\beta 1$-induced fibrosis to identify additional precise targets. It has been demonstrated that IncRNAs can directly regulate some biological processes in a diseaseand tissue-specific manner (6). For example, the lncRNA SARCC binds to and destabilizes the androgen receptor post-transcriptionally to regulate its expression, which can contribute to tumor progression in renal cell carcinoma (17). Targeting lncRNAs may therefore provide a novel therapeutic approach in various types of cancer. Results from the present study demonstrated that uc.412 was upregulated in MCs following TGF- $\beta 1$ treatment and that uc.412 overexpression significantly increased MC proliferation. It is therefore crucial to understand the underlying mechanism of uc.412 in the regulation of MC proliferation and to determine whether uc.412 may be considered as a therapeutic target in mesangial proliferative kidney diseases.

To determine the potential biological functions of uc. 412 in MCs, DEGs were identified between MCs that overexpressed uc.412 and negative control MCs using high-throughput RNA sequencing. The results from this analysis identified 1,305 DEGs, including 462 up- and 843 downregulated genes.

The number of samples analyzed in the present study was limited, criteria for selecting DEGs was set as $\mid \mathrm{FCl}$ $\geq 1.5$. In order to verify the RNA-sequencing results, expression levels of five upregulated and 4 downregulated DEGs were subsequently verified by RT-qPCR. Serum secretory phospholipase A2-IIa, which is encoded by PLA2G2A, has been demonstrated to be associated with cell signaling, apoptosis, remodeling of cell membranes and inflammatory responses (16). The $\beta$-catenin-dependent Wnt signaling pathway may regulate PLA2G2A expression through the 
A

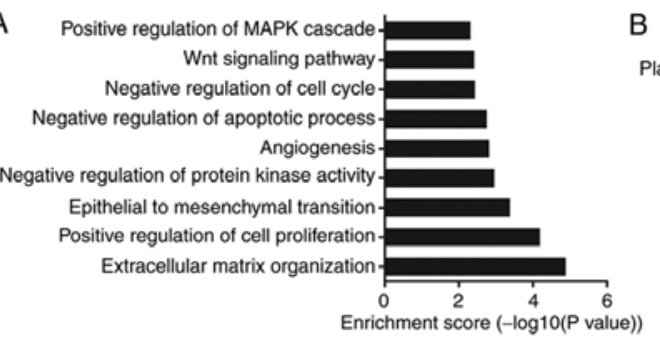

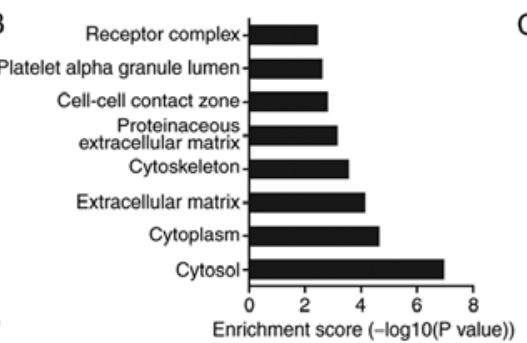

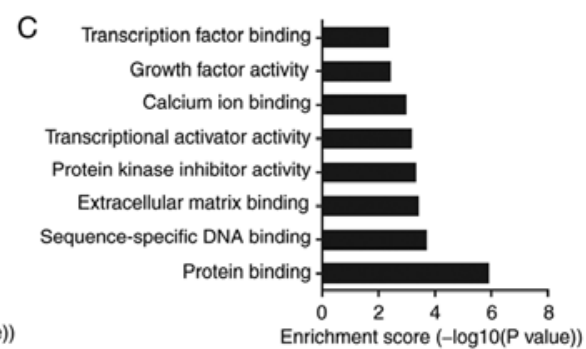

Figure 4. GO term analysis of the identified DEGs. (A-C) The 1,305 DEGS identified between uc.412 overexpressing mesangial cells and control cells were analyzed for GO term enrichment, including (A) biological processes, (B) cellular components and (C) molecular functions. GO, gene ontology; MAPK, mitogen-activated protein kinase; uc.412, long non-coding RNA uc.412.

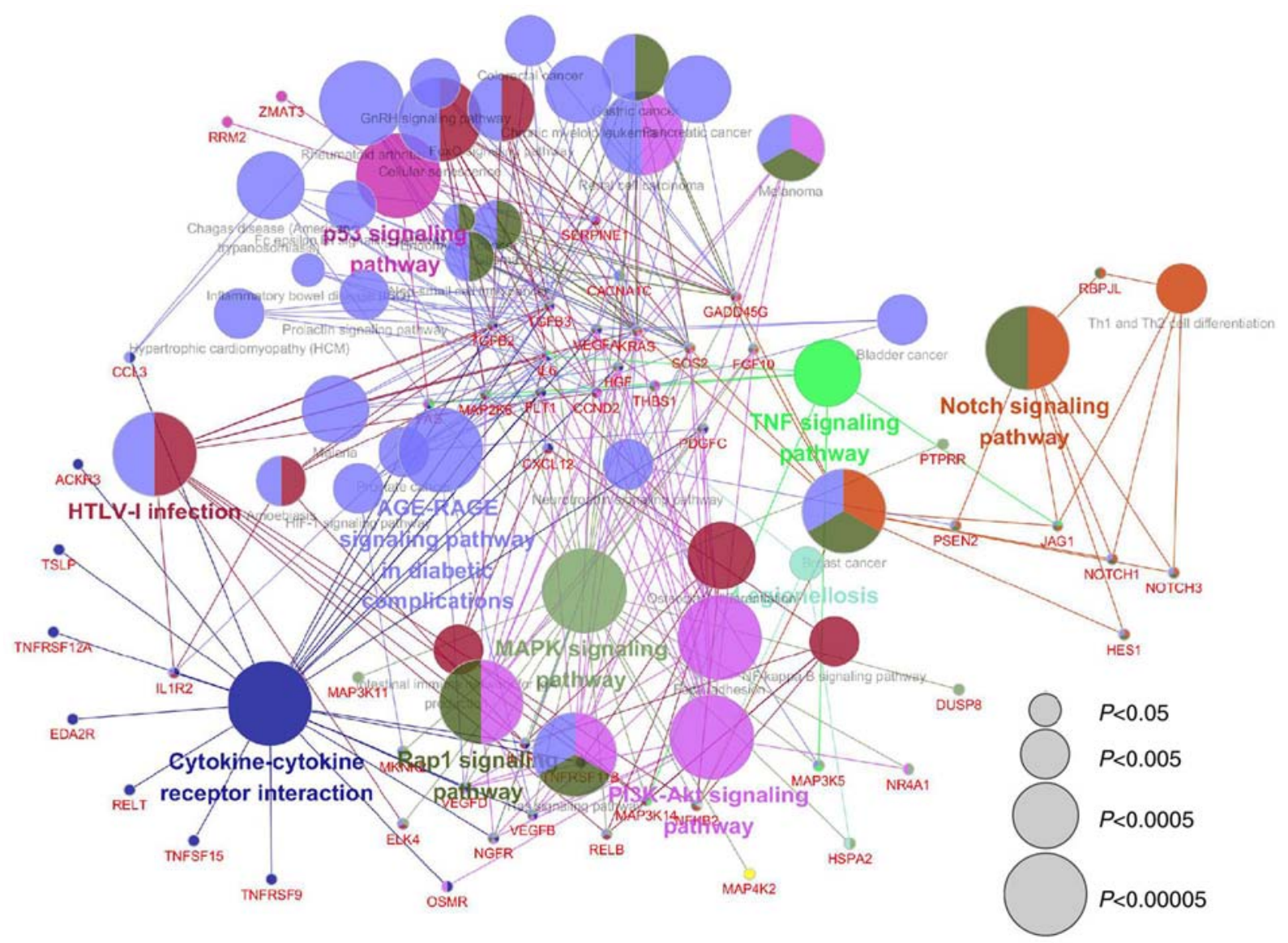

Figure 5. Analysis of the potential target genes of uc.412 by Kyoto Encyclopedia of Genes and Genomes pathway analysis.

$\beta$-catenin-dependent Wnt signaling pathway; the Wnt pathway is involved in numerous kidney diseases, including glomerular diseases, ischemic kidney injury, interstitial fibrosis, diabetic nephropathy and cystic kidney diseases (18). Deregulation of the Wnt pathway has been associated with fibrotic diseases in kidney (19). Numerous molecules have been demonstrated to be associated with the establishment of the fibrotic condition, including angiotensin II, TGF- $\beta 1$ and CTGF (19). Furthermore, a crosstalk between the Wnt pathway and these signaling molecules has been identified (19). uc.412 may therefore regulate the expression of certain genes involved in proliferation and fibrosis of MCs and may serve a role in the pathways that contribute to the development of kidney diseases.
The results from GO analysis indicated that the identified DEGs may be involved in certain biological process, which may serve crucial roles in the incidence and development of certain kidney diseases. For example, podocyte injury may lead to glomerulosclerosis in IgA nephropathy (IgAN) (19). Various factors contributing to epithelial-to-mesenchymal transition (EMT) have been demonstrated to be responsible for podocyte damage. For example, hyperglycemia has been demonstrated to induce podocyte EMT through TGF- $\beta /$ Smad classic pathway, the Wnt/ $\beta$-catenin signaling pathway, the integrin/integrin-linked kinase signaling pathway, the mitogen-activated protein kinase signaling pathway, the Jagged/Notch signaling pathway and the nuclear factor- $\kappa \mathrm{B}$ signaling pathway and contribute to podocyte 
damage (20). However, it was previously reported that the PI3K/AKT-signaling pathway may be involved in EMT in podocytes following treatment with mesangial medium from cells isolated from IgAN patients (19). In addition, previous studies reported that directly modulating cell cycle progress could regulate RMC proliferation $(21,22)$. Furthermore, immunosuppressive agents could affect the cell cycle progression, apoptosis and proliferation of MCs (23). Some kidney diseases can be ameliorated through cell cycle-dependent mechanisms (23); for example, MC cell cycle can be regulated using rapamycin to induce an inhibition of $\mathrm{MC}$ proliferation, a reduction of IgA deposition and a slow IgAN progression (24). Results from the present study indicated that uc.412 downstream genes may regulate cell cycle, which indicated that uc.412 may be involved in MC proliferation.

The results from KEGG clustering analysis, identified several key pathways and a complex regulatory network. It has been reported that p53 is upregulated in a mouse model of Habu nephritis, which suggested that p53 can modulate MC proliferation and apoptosis (25). Furthermore, Notch signaling pathway could activate TGF- $\beta$ to accelerate the development of diabetic nephropathy (26). The dysregulation of Notch signaling molecules has been reported to serve a vital role in fibrosis models, renal injuries and diabetic kidney biopsies (26). For example, Jagged1, which is the canonical Notch ligand, is upregulated in a TGF- $\beta$ dependent manner during chronic kidney diseases (26). The present study therefore hypothesized that uc.412 may serve a crucial role in the development of mesangial proliferative glomerulonephritis.

STRING was used to construct PPI networks from RNA sequencing data. The results demonstrated that SERPINE1 interacted with a number of proteins in the upregulated genes PPI network. For example, SERPINE1 may interact with CTGF, which has been demonstrated to serve a crucial role in the progression of kidney fibrosis, as it participates in cell migration, proliferation and inflammation (27). CTGF can also stimulate MCs to synthesize more fibronectin and collagen type I (28). MMP3 was also revealed to interact with a number of proteins in the downregulated genes PPI network. In addition, a series of genes associated with cell proliferation were involved in the interaction networks. For example, Notch3 interacted with cyclin D2, dynamin 3 and EPH receptor A7 (29). Downregulating cellular expression of Notch3 may therefore improve cell proliferation (29). In conclusion, the results from the present study exhibited an interlaced transcript network associated with uc.412, which may have important effects on $\mathrm{MC}$ proliferation.

Identifying the role of uc.412 in MC proliferation is crucial to understanding certain glomerular diseases. The present study identified the downstream genes of uc.412 and a complicated mesangial cell network. These results may aid in developing novel therapeutic strategies to limit the pathological process of glomerular diseases associated with uncontrolled MC proliferation. However, the underlying mechanism and biological function of uc.412 in the regulation of MC proliferation require further investigation.

\section{Acknowledgements}

Not applicable.

\section{Funding}

The present study was supported by The National Natural Science Foundation of China (grant no. 81670650), The Natural Science Foundation of Jiangsu Province (grant no. BK20161071), The Project of Nanjing Medical Youth Talent (grant no. QRX17106), The Project of Jiangsu Provincial Maternal and Child Talent (grant no. FRC201737), The Research Project of Jiangsu Provincial Health Commission (grant no. LGY2018071) and The Science and Technology Development Foundation of Nanjing Medical University (grant no. 2016NJMU036).

\section{Availability of data and materials}

The datasets used and/or analyzed during the present study are available from the corresponding author on reasonable request.

\section{Authors' contributions}

SL, AZ, BW and WG conceived and designed the study. MY, ZG and XW performed the experiments. HS, GQ, XL, QF and $\mathrm{XZ}$ interpreted the data. MY wrote the manuscript. AZ critically reviewed and revised the manuscript. SL and QF revised the manuscript. All authors have read and approved the final version of the manuscript.

\section{Ethics approval and consent to participate}

The present study was approved by the Medical Ethics Committee of the Second Affiliated Hospital of Nanjing Medical University (Nanjing, China; approval number: 2016KY008-01).

\section{Patient consent for publication}

Not applicable.

\section{Competing interests}

The authors declare that they have no competing interests.

\section{References}

1. Cove-Smith A and Hendry BM: The regulation of mesangial cell proliferation. Nephron Exp Nephrol 108: e74-e79, 2008.

2. Kurogi Y: Mesangial cell proliferation inhibitors for the treatment of proliferative glomerular disease. Med Res Rev 23: 15-31, 2003.

3. Zhang A, Han Y, Wang B, Li S and Gan W: Beyond gap Junction channel function: The expression of $\mathrm{Cx} 43$ contributes to aldosterone-induced mesangial cell proliferation via the ERK1/2 and PKC pathways. Cell Physiol Biochem 36: 1210-1222, 2015.

4. Loeffler I and Wolf G: Transforming growth factor- $\beta$ and the progression of renal disease. Nephrol Dial Transplant 29 (Suppl 1): i37-i45, 2014.

5. Rodrigues-Diez R, Rayego-Mateos S, Orejudo M, Aroeira LS, Selgas R, Ortiz A, Egido J and Ruiz-Ortega M: TGF-beta blockade increases renal inflammation caused by the C-terminal module of the CCN2. Mediators Inflamm 2015: 506041, 2015.

6. Kopp F and Mendell JT: Functional classification and experimental dissection of long noncoding RNAs. Cell 172: 393-407, 2018.

7. Tang PM, Tang PC, Chung JY and Lan HY: TGF- $\beta 1$ signaling in kidney disease: From Smads to long non-coding RNAs. Noncoding RNA Res 2: 68-73, 2017. 
8. Tang W, Zhang D and Ma X: RNA-sequencing reveals genome-wide long non-coding RNAs profiling associated with early development of diabetic nephropathy. Oncotarget 8: 105832-105847, 2017.

9. Bai X, Geng J, Li X, Wan J, Liu J, Zhou Z and Liu X: Long noncoding RNA LINC01619 regulates MicroRNA-27a/Forkhead box protein $\mathrm{O} 1$ and endoplasmic reticulum stress-mediated podocyte injury in diabetic nephropathy. Antioxid Redox Signal 29: 355-376, 2018.

10. Qu L, Ding J, Chen C, Wu ZJ, Liu B, Gao Y, Chen W, Liu F, Sun W, Li XF, et al: Exosome-transmitted lncARSR promotes sunitinib resistance in renal cancer by acting as a competing endogenous RNA. Cancer Cell 29: 653-668, 2016.

11. Zhang A, He Y, Wang B, Shi H and Gan W: Analysis of the differential expression of long noncoding RNAs in experimental mesangial cells proliferation induced by TGF- $\beta$. Chin J Nephrol 31: 774-779, 2015.

12. Livak KJ and Schmittgen TD: Analysis of relative gene expression data using real-time quantitative PCR and the 2(-Delta Delta C(T)) method. Methods 25: 402-408, 2001.

13. Ma F, Fuqua BK, Hasin Y, Yukhtman C, Vulpe CD, Lusis AJ and Pellegrini M: A comparison between whole transcript and 3'RNA sequencing methods using Kapa and Lexogen library preparation methods. BMC Genomics 20: 9, 2019

14. Huang da W, Sherman BT and Lempicki RA: Bioinformatics enrichment tools: Paths toward the comprehensive functional analysis of large gene lists. Nucleic Acids Res 37: 1-13, 2009.

15. Huang da W, Sherman BT and Lempicki RA: Systematic and integrative analysis of large gene lists using DAVID bioinformatics resources. Nat Protoc 4: 44-57, 2008.

16. Cui Y, Huang Y, Wu X, Zheng M, Xia Y, Fu Z, Ge H, Wang S and Xie H: Hypoxia-induced tRNA-derived fragments, novel regulatory factor for doxorubicin resistance in triple-negative breast cancer. J Cell Physiol 234: 8740-8751, 2019.

17. Zhai W, Sun Y, Jiang M, Wang M, Gasiewicz TA, Zheng J and Chang C: Differential regulation of LncRNA-SARCC suppresses VHL-mutant RCC cell proliferation yet promotes VHL-normal RCC cell proliferation via modulating androgen receptor/HIF-2 $\alpha / \mathrm{C}-\mathrm{MYC}$ axis under hypoxia. Oncogene 35 4866-4880, 2016.

18. Dong T, Peng Y, Zhong N, Liu F, Zhang H, Xu M, Liu R, Han M, Tian X, Jia J, et al: Perfluorodecanoic acid (PFDA) promotes gastric cell proliferation via SPLA2-IIA. Oncotarget 8 : 50911-50920, 2017.
19. Kawakami T, Ren S and Duffield JS: Wnt signalling in kidney diseases: Dual roles in renal injury and repair. J Pathol 229: 221-231, 2013.

20. Ying Q and Wu G: Molecular mechanisms involved in podocyte EMT and concomitant diabetic kidney diseases: An update. Ren Fail 39: 474-483, 2017.

21. Lan T, Wu T, Chen C, Chen X, Hao J, Huang J, Wang L and Huang $\mathrm{H}$ : Berberine attenuates high glucose-induced proliferation and extracellular matrix accumulation in mesangial cells: Involvement of suppression of cell cycle progression and NF-кB/AP-1 pathways. Mol Cell Endocrinol 384: 109-116, 2014.

22. Chen D, Li Y, Mei Y, Geng W, Yang J, Hong Q, Feng Z, Cai G, Zhu $\mathrm{H}$, Shi $\mathrm{S}$, et al: miR-34a regulates mesangial cell proliferation via the PDGFR- $\beta$ /Ras-MAPK signaling pathway. Cell Mol Life Sci 71: 4027-4042, 2014

23. Zhou X, Workeneh B, Hu Z and Li R: Effect of immunosuppression on the human mesangial cell cycle. Mol Med Rep 11: 910-916, 2015.

24. Tian J, Wang Y, Liu X, Zhou X and Li R: Rapamycin ameliorates IgA nephropathy via cell cycle-dependent mechanisms. Exp Biol Med (Maywood) 240: 936-945, 2015.

25. Lu Y, Wen J, Chen D, Wu L, Li Q, Xie Y, Wu D, Liu X and Chen X: Modulation of cyclins and $\mathrm{p} 53$ in mesangial cell proliferation and apoptosis during Habu nephritis. Clin Exp Nephrol 20: 178-186, 2016.

26. Liu L, Gao C, Chen G, Li X, Li J, Wan Q and Xu Y: Notch signaling molecules activate TGF- $\beta$ in rat mesangial cells under high glucose conditions. J Diabetes Res 2013: 979702, 2013.

27. Toda N, Mukoyama M, Yanagita M and Yokoi H: CTGF in kidney fibrosis and glomerulonephritis. Inflamm Regen 38: 14, 2018.

28. Riser BL, Denichilo M, Cortes P, Baker C, Grondin JM, Yee J and Narins RG: Regulation of connective tissue growth factor activity in cultured rat mesangial cells and its expression in experimental diabetic glomerulosclerosis. J Am Soc Nephrol 11: 25-38, 2000.

29. Zhao WX, Zhuang X, Huang TT, Feng R and Lin JH: Effects of Notch 2 and Notch 3 on cell proliferation and apoptosis of trophoblast cell lines. Int J Med Sci 12: 867-874, 2015.

This work is licensed under a Creative Commons Attribution-NonCommercial-NoDerivatives 4.0 International (CC BY-NC-ND 4.0) License. 